Extraction Chromatography of Neodymium

by an Organophosphorous Extractant

Supported on Various Polymeric Resins

Doreen Y. Takigawa 


\title{
EXTRACTION CHROMATOGRAPHY OF NEODYMIUM BY AN ORGANOPHOSPHOROUS EXTRACTANT SUPPORTED ON VARIOUS POLYMERIC RESINS
}

by

Doreen Y. Takigawa

\begin{abstract}
Fifteen resins coated with dihexyl-N,N-diethylcarbamoylmethylphosphonate (CMP) were studied for their extraction of neodymium ( $\mathrm{Nd}$ ) in 4.0 and $7.0 \mathrm{M}$ nitric acid. Resin properties, such as chemical composition and physical morphology. which can influence Nd extraction as well as subsequent resin regeneration (Nd stripping), were identified. Hydrophilic or polar resins coated with CMP efficiently extracted the Nd. Resins initially washed free of residual monomer and solvent before CMP coating outperformed their untreated counterparts. The macroporous styrene-divinylbenzene hydrophobic resins that were high in surface area were less effective supports compared with hydrophilic microporous Aurorez, polybenzimidazole (PBI) and macroporous Amberlite polyacrylic resins. Only one resin, Duolite C-467, showed no measurable improvement in Nd extraction with CMP coating. CMP-coated Aurorez PBI, a microporous and hydrophilic polymeric resin with an average surface area, showed the best overall efficiency for $\mathrm{Nd}$ removal and resin regeneration.
\end{abstract}

\section{INTRRODUCTION}

Scientists at Los Alamos National Laboratory are investigating extraction chromatography as a viable method for recovering actinides from high-acid process effluents. ${ }^{1}$ In this method synthetic resins act as polymeric adsorbents for a bidentate organophosphorous extractant specific for the removal of actinides. Extraction chromatography studies reported in the literature describe the removal of copper by LIX extractants. ${ }^{2}$ Other reports describe the separation of americium from plutonium by a sequential procedure consisting of anion exchange and extraction chromatography. ${ }^{3}$ Navratil ${ }^{4}$ reports the use of non-ionic macroporous resins for the removal of dissolved extractants, such as organophosphorous compounds from aqueous waste streams.

The objective of the work reported here was to identify, by evaluating a variety of commercial resins coated with dihexyl-N,N-diethylcarbamoylmethylphosphonate (CMP), the effects of resin composition and physical morphology on neodymium (Nd) extraction. $\mathrm{Nd}$ served as an analog of americium, an actinide commonly found in acid waste streams. An understanding of the significant factors influencing the extraction behavior of the coated polymer resin is likely to lead to the development of a new generation of improved resins. Such resins will consist of extractants covalently bound on those polymer resins shown by experiment to be most suitable for this use.

\section{EXPERIMENTAL METHOD}

\section{Reagents}

All reagents were analytical grade products. CMP purchased from Industrial and Specialty Chemicals (Niagara Falls, New York) was used without further purification. Stock solutions of 
$2.0 \mathrm{~g} / \mathrm{l} \mathrm{Nd}$ were made from $\mathrm{Nd}$ nitrate hexahydrate (from the Aldrich Chemical Co.) in $4.0 \mathrm{M}$ or $7.0 \mathrm{M}$ nitric acid solutions. Buffer solutions for $\mathrm{Nd}$ analysis were prepared from sodium acetate and acetic and nitric acid and were stabilized at a pH of 3.3. Arsenazo III (also from the Aldrich Chemical $\mathrm{Co}$.) in solution was used to obtain a color reaction with $\mathrm{Nd}$ to allow spectrophotometric determination of $\mathrm{Nd}$ at very low concentrations.

\section{Polymeric Adsorbent Resins}

- Aurorez polybenzimidazole (PBI) resin in two mesh sizes, 150 to $250 \mu \mathrm{m}$ and 250 to $500 \mu \mathrm{m}$, were obtained from Celanese Corporation.

- Biobeads SM-2 and SM-7 were supplied by Biorad Laboratories.

- Amberlite IRC: 50S, 76, and 718; Amberlite XAD: 2, 4, 7, 8, and 16; and Duolite: C-467 and S-761 were obtained from Rohm and Haas Company.

- Lewatit OC A062 was obtained fron Mobay Chemical Company.

- Dianex HP 20 was obtained from Dianex Systems.

\section{Resin-Coating Procedure}

A mixture of $5 \mathrm{ml}$ of CMP and $2 \mathrm{~g}$ of resin was shaken at $350 \mathrm{rpm}$ for $60 \mathrm{~min}$ in a temperature-controlled shaker bath at $55^{\circ} \mathrm{C}$. The CMP was filtered from the resin, and the resin was rinsed and filtered repeatedly with a total of $500 \mathrm{ml}$ of deionized water. This assured removal of any residual CMP adsorbed on the surface; CMP can adversely affect spectrophotometric evaluation of $\mathrm{Nd}$.

\section{Nd Extraction Experiment}

The coated resin beads were divided by equal weights into two separate vials. To one vial, $5 \mathrm{ml}$ of a Nd stock solution containing $2.0 \mathrm{~g} / \mathrm{l} \mathrm{Nd}$ in $4.0 \mathrm{M} \mathrm{HNO}_{3}$ was added, and to the other vial, $5 \mathrm{ml}$ of a stock solution containing $2.0 \mathrm{~g} / \mathrm{Nd}$ in $7.0 \mathrm{M} \mathrm{HNO}_{3}$ was added. These vials were placed in a $40^{\circ} \mathrm{C}$ temperature-controlled bath and agitated for $10 \mathrm{~min}$ at $350 \mathrm{rpm}$. The 10 -min batch contact time was selected to simulate a practical kinetic evaluation of the resin in a column.

The Nd remaining in the solution was evaluated by ultraviolet spectrophotometry, to determine, by difference, the amount of $\mathrm{Nd}$ adsorbed by the resin. Analysis of the $\mathrm{Nd}$ was performed by complexing the $\mathrm{Nd}$ with Arsenazo III (2:1 molar ratio Arsenazo: Nd) at a $\mathrm{pH}$ of 3.5. The solution containing $\mathrm{Nd}$ was diluted to concentrations in the $1 \times 10^{-5} \mathrm{M}$ to $5 \times 10^{-5} \mathrm{M} \mathrm{Nd}$ concentration region, where Beer's law for Nd/Arsenazo complex was obeyed at $\lambda_{\max }=658 \mathrm{~nm}$. In every experiment, the $\mathrm{Nd}$ stock solution was also analyzed with the same dilution and Arsenazo complexation technique to establish the $\mathrm{Nd}$ standard base line.

\section{Nd-Stripping Experiment}

The resin tested for $\mathrm{Nd}$ extraction was filtered free from the $\mathrm{Nd}$ solution and placed in a vial. To this resin was added $3.5 \mathrm{ml}$ of $0.1 \mathrm{M} \mathrm{HNO}_{3}$. This mixture was agitated for $1 \mathrm{~min}$ at $350 \mathrm{rpm}$ in a temperature-controlled shaker bath at $40^{\circ} \mathrm{C}$. The amount of $\mathrm{Nd}$ stripped from the resin was evaluated spectrophotometrically by the procedure described above. 


\section{RESULTS AND DISCUSSION}

The 15 resins coated with CMP and tested for Nd extraction are listed in Table 1 . The resins are ranked according to their overall performance for $\mathrm{Nd}$ extraction (in 4.0 and $7.0 \mathrm{M}$ $\mathrm{HNO}_{3}$ ) and their subsequent stripping and resin-regeneration ability. The ratings for each resin were assigned according to their comparative rankings on extraction and stripping abilities in $\mathrm{Nd}$ test solutions at 4.0 and $7.0 \mathrm{M} \mathrm{HNO}_{3}$. Tables 2 and 3 rank the resins according to their $\mathrm{Nd}$ extraction ability in $4.0 \mathrm{M} \mathrm{HNO}_{3}$ and $7.0 \mathrm{M} \mathrm{HNO}_{3}$, respectively.

\section{PBI}

The Aurorez PBI resin is a microporous spherical polymer having the following physical properties in the dry $\operatorname{state}^{5}$ : bead size, $150 \mu \mathrm{m}$; pore size, $80 \AA$; specific surface area, 20 to $35 \mathrm{~m}^{2} / \mathrm{g}$; and $5 \%$ to $10 \%$ swelling in water. The resin was supplied wet with a moisture content of approximately $80 \%(w / w)$. The PBI repeating unit structure,

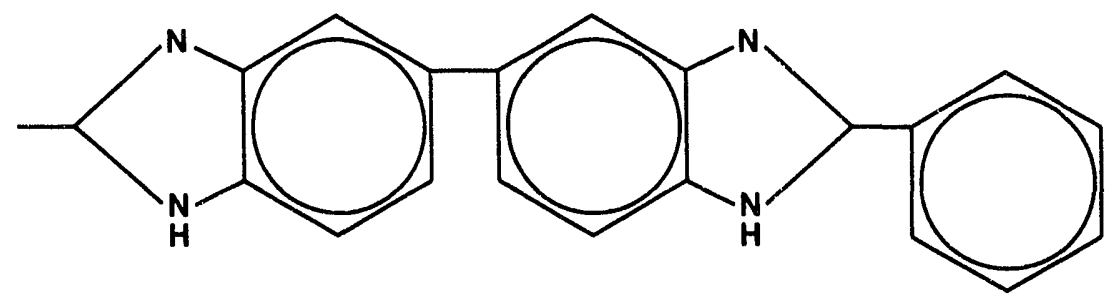

has two tertiary nitrogens amenable to protonation, with a theoretical capacity of $6.5 \mathrm{meq} / \mathrm{g}$. PBI is an acid-, temperature-, and radiation-resistant hydrophilic polymer that has been considered for use in ion exchange, in gas and liquid chromatography, and as an inert substrate for oxidizing and reducing agents. Although Aurorez PBI without CMP coating extracted no Nd, when coated with CMP, it extracted very well (Tables 2 and 3 ). This finding suggests that PBI adsorbs CMP, which in turn creates a favorable environment for the sorption of $\mathrm{Nd}$. The hydrophilic/polar nature of PBI resin appears to enhance the diffusion of metals in an aqueous environment.

The sorption of $\mathrm{PBI}$ for CMP is not surprising. PBI efficiently removes organic compounds such as phenols and carboxylic acids. ${ }^{6}$ Studies have found a sharp increase in the sorption capacity with the length of the carbon chain in a series of carboxylic acids. Thus, apolar (or hydrophobic) bonding interactions between the PBI and hydrocarbon components of the longchain carboxylic acids enhances sorption. Hydrogen-bonding interactions also appear to play a role in the enhanced sorption of the phenols. Similar interactions have presumably occurred during the CMP sorption onto PBI observed in the work reported here.

\section{Amberlite IRC-76}

Unlike PBI, Amberlite IRC-76 is a weakly acidic macroporous resin supplied in the hydrogen form. It has carboxylic acid functional-exchange sites within a cross-linked polyacrylic matrix. The physical properties of Amberlite IRC-76 are as follows: bead size, 16 to 50 mesh (290 to $1000 \mu \mathrm{m}) ; 54 \%$ to $58 \%$ moisture content; and macroporous. Because Amberlite IRC-76 is supplied in the hydrogen form, it is effective in removing ions associated with carbonate alkalinity as well as the adsorption of basic compounds such as CMP. Such macroreticular resins will adsorb significant quantities of compatible compounds, filling in the available voids. Also, the hydrophilic polyacryiic matrix of Amberlite IRC-76 is similar to that of Aurorez PBI and may enhance the diffusion of metals in an aqueous environment. As indicated in Tables 2 anc 3. Amberlite IRC-76/CMP behaves like Aurorez PBI/CMP in its extraction capabilities at 4.0 and 7.0 $\mathrm{M} \mathrm{HNO}_{3}$, but its stripping abilities are considerably better. 
Table 1. Overall Rating of CMP-Coated Resins

\begin{tabular}{lc}
\hline Resin & Rating \\
\hline Aurorez PBI & 4.5 \\
Amberlite IRC-76 & 5.83 \\
SM-7 & 6.00 \\
Lewatit OC A062 & 7.00 \\
XAD-8 & 7.17 \\
Dianex HP 20 & 7.17 \\
XAD-7 & 7.5 \\
Duolite S761 & 7.67 \\
XAD-16 & 11.33 \\
XAD-4 & 11.67 \\
XAD-2 & 12.0 \\
Amberlite IRC-718 & 12.34 \\
\hline \hline
\end{tabular}

Table 2. Nd Extraction/Stripping from CMPCoated Resins at 4.0 $\mathrm{M} \mathrm{HNO}_{3}$

\begin{tabular}{lcc}
\hline Resin & Extraction (\%) & Stripping (\%) \\
\hline Duolite C-467 & 88.4 & 8.3 \\
SM-7 & 87.1 & 28.9 \\
Aurorez PBI & 83.2 & 45.3 \\
XAD-7 & 83.1 & 17.1 \\
Amberlite IRC-76 & 82.3 & 52.6 \\
Dianex HP 20 & 81.7 & 36.9 \\
XAD-8 & 78.0 & 51.2 \\
Duolite S-761 & 76.7 & 37.3 \\
IRC 50-S & 75.4 & 61.2 \\
Lewatit OC A062 & 71.4 & 50.8 \\
XAD-4 & 71.1 & 27.2 \\
XAD-16 & 66.3 & 41.4 \\
SM-2 & 60.1 & 58.5 \\
XAD-2 & 46.3 & 50.6 \\
Amberlite IRC-718 & 32.3 & 63.1 \\
\hline \hline
\end{tabular}

Table 3. Nd Extraction/Stripping from CMPCoated Resins at 7.0 $\mathrm{M} \mathrm{HNO}_{3}$

\begin{tabular}{lcc}
\hline Resin & Extraction (\%) & Stripping (\%) \\
\hline SM-7 & 92.4 & 20.9 \\
Aurorez PBI & 88.5 & 37.7 \\
Lewatit OC A062 & 86.2 & 39.0 \\
Duolite S-761 & 86.0 & 31.9 \\
XAD-7 & 85.8 & 12.0 \\
Duolite C-467 & 85.8 & 10.2 \\
IRC-76 & 85.5 & 50.6 \\
XAD-8 & 84.5 & 36.1 \\
Dianex HP 20 & 80.0 & 34.3 \\
XAD-16 & 79.3 & 41.4 \\
Amberlite IRC-50S & 78.8 & 75.8 \\
XAD-4 & 75.5 & 26.5 \\
SM-2 & 58.8 & 46.6 \\
XAD-2 & 57.8 & 40.9 \\
Amberlite IRC-718 & 33.7 & 72.0 \\
\hline \hline
\end{tabular}




\section{SM-7, XAD-7, and XAD-8}

The chemical composition of the three macroporous resins, SM-7, XAD-7, and XAD-8, is the same as that of an acrylic, nonfunctionalized polymer matrix. In fact, SM-7 and XAD-7 are the same resin (20 to 50 mesh, $90-\AA$ pore size, and $450-\mathrm{m}^{2} / \mathrm{g}$ surface area). Biorad Laboratories, which supplies SM-7, acknowledged that the XAD-7 resin they purchase from Rohm and Haas is rigorously washed with a mixture of hot water, $70 \%$ isopropanol, and $100 \%$ methanol. This technique presumably washes the beads free of excess toluene, cyclohexanol, methyl methacrylate, and ethylene dimethacrylate, which are left as surface residue from the polymerization. As indicated in Tables 1 through 3, SM-7/CMP and XAD-7/CMP behave differently. SM-7/CMP outperforms XAD-7/CMP in Nd extraction and stripping in 4.0 and $7.0 \mathrm{M} \mathrm{HNO}_{3}$ test solutions. XAD-8, which is lower in surface area-160 m²/g-but larger in pore size $-225 \mathrm{~A}$-performs similarly to the XAD-7 in extraction of $\mathrm{Nd}$ but better in stripping.

\section{Duolite C-467}

The Duolite C-467 resin is a macroporous, divinylbenzene cross-linked polystyrene with aminophosphonic functional groups. The bead size is 0.3 to $1 \mathrm{~mm}$ (18 to 50 mesh size). The Nd extraction and stripping properties before and after coating with CMP are the same. Duolite C-467 is the only resin tested that extracted $\mathrm{Nd}$ in $4.0 \mathrm{M}$ and $7.0 \mathrm{M} \mathrm{HNO}_{3}$ to the same degree, regardless of whether it was coated with CMP. Although the polymer matrix remained the same, the aminophosphonic functionality enhanced the adsorption of $\mathrm{Nd}$ considerably more than did the nonfunctionalized polystyrene. Because this resin is such a strong complexant for $\mathrm{Nd}$, however, its stripping property is very poor (Tables 2 and 3 ).

\section{Lewatit OC A062 and Dianex HP 20}

Both Lewatit OC A062 and Dianex HP 20 are macroporous and macroreticular polystyrene divinylbenzene. Lewatit OC A062 has the following properties: 40 mesh size, $110-\AA$ pore size, and $500-\mathrm{m}^{2} / \mathrm{g}$ surface area. Dianex HP 20 has a mesh size of 20 to 50 and a surface area of $511 \mathrm{~m}^{2} / \mathrm{g}$. These two macroporous resins are prepared differently from the polystyrene divinylbenzene XAD-2, -4 , and -16 series described below. ${ }^{7}$ The resin synthesis involves copolymerization of styrene and divinylbenzene in the presence of an inert polymer. When the polymerization is complete, the occluded polymer is extracted, resulting in a resin with a uniform pore-size distribution. Macroreticular resins have a definitive and permanent internal porous structure that can absorb significant quatitites of solvent, filling the available void space. Comparison of the Nd extraction results for these resins over the XAD series suggests that macroporous and macroreticular resins may adsorb more CMP, resulting in enhanced adsorption for $\mathrm{Nd}$.

\section{Duolite S-761}

The physical properties of Duolite S-761 resin are as follows: 16 to 50 mesh size, $600-\AA$ pore size, and $300-\mathrm{m}^{2} / \mathrm{g}$ surface area. The phenolic composition of this resin would make it intermediate in polarity between the polar acrylic XAD-7 and the apolar styrene XAD-2, -4 , and -16 series adsorbents. Accordingly, the effect of this composition is reflected in Table 1, where the overall performance falls between those of the acrylic and the styrene resins. 


\section{Amberlite IRC-50S}

Amberlite IRC-50S, a weakly acidic, macroporous, cation-exchange resin is prepared from methacrylic acid and divinylbenzene. The carboxylic functionality has an affinity for basic compounds, hence the adsorption for CMP. As observed in Tables 2 and 3, its Nd extraction ability in $4.0 \mathrm{M}$ and $7.0 \mathrm{M} \mathrm{HNO}_{3}$ was lower than that of IRC-76, which is the strictly hydrophilic cation-exchange resin (with hydrophilic cross links) composed of a polyacrylic ester matrix. Amberlite IRC-50S, however, outperformed the nonfunctionalized polystyrene divinylbenzene series XAD-2, -4 , and -16 in Nd extraction and, more significantly, in its efficient stripping ability.

\section{SM-2 and XAD-2, -4 , and -16}

The SM-2 and XAD-2, -4 , and -16 resins are all polystyrene divinylbenzene in composition. The SM-2 resin is exactly the same as the XAD-2 resin in physical structure: 20 to 60 mesh, $90-\AA$ pore size, and $300-\mathrm{m}^{2} / \mathrm{g}$ surface area. The SM-2 resin, however, outperforms the XAD-2 resin, most likely for the same reason that SM-7 outperforms XAD-7. The superior performance of SM-2 and SM-7 is attributed to the cleaning of surface residue left over from the polymerization. As indicated in Table 1, XAD-16 performs better than XAD-4, which performs better than XAD-2. This performance order corresponds to increases in respective surface areas: XAD-16, -4 , and -2 have surface areas of 800,725 , and $300 \mathrm{~m}^{2} / \mathrm{g}$, respectively.

\section{Amberlite IRC-718}

Amberlite IRC-718 is a cation-exchange resin with iminodiacetic acid functicnality bonded to a styrene divinylbenzene matrix. This resin has high affinity for heavy-metal ions at $\mathrm{pH}$ greater than 1.5. However, in most cases, the resin's capazity for metal ions falls off sharply at low $\mathrm{pH}$. The adsorption of CMP and the concomitant extraction of Nd onto IRC-718 may be small because of the hydrophobic matrix. As with the other carboxylic functionalities, the Nd stripping ability is relatively high compared with that of nonfunctionalized or aminophosphonic-functionalized resins.

\section{CONCLUSIONS}

Hydrophilic and polar resins coated with CMP exhibited the best extraction behavior for Nd. Biobeads SM-2 and SM-7 showed that resins that are extensively washed free of residual monomer and solvent before CMP coating outperform thrir unwashed counterparts XAD-2 and XAD-7 obtained from Rohm and Haas. Although hydrophobic polystyrene divinylbenzene nonfunctionalized adsorbents do not perform nearly as well as hydrophilic resins, when compared with each other, the more macroporous and higher-surface-area polystyrene-based resin performed better. Duolite C-467, a polystyrene-based resin with aminophosphonic functional groups, extracted $\mathrm{Nd}$ well by itself and showed no enhanced Nd extraction with CMP coating. Duolite C-467 is such a strong complexant, however, that its Nd-stripping ability is very poor. Aurorez PBI, a microporous resin prepared from a hydrophilic, linear aromatic polymer performed the best when coated with CMP. As an acid- and radiation-resistant polymer, PBI is well suited for its use in actinide recovery operations. Because PBI has two secondary nitrogens, which can become functionalized, it may be developed into an exceptional resin bearing covalently bound, actinide-specific extractants. Work is currently being pursued in this area, with the attachment of malonamide extractants to PBI. 


\section{ACKNOWLEDGMENTS}

The author gratefully acknowledges the contributions of Harry Bush and A. James Gray.

\section{REFERENCES}

1. S. L. Yarbro, S. L. Dunn, and S. B. Shreiber, "Processing Low Level Radioactive Solutions using Extraction Chromatography," 1989 AIChE Annual Meeting, San Francisco, California, 1989.

2. M. Hemmes and J. R. Parrish. "Extraction Chromatography of Copper by LIX-64N and LIX-65N Supported on the Macroporous Resin. XAD-7," Analytica Chimica Acta 94, 307 (1977).

3. M. Yamamoto, K. Komura, and N. Sakanoue, "A Simple Sequential Separation Method of Pu and Am by Anion Exchange and Extraction Chromatography," Radiochimica Acta 29, 205 (1981).

4. J. D. Navratil, "Removal of Solubilized Sulvent Extractants from Aqueous Waste Streams," J. Nuc. Sci. and Tech. 18(7), 561 (1981).

5. A. Buckley, D. E. Stuetz, and G. A. Serad, "Polybenzimidazoles," Encyclopedia of Polym. Sci. and Eng., Vol. 11, p. 572, (John Wiley \& Sons, Inc., New York, 1988).

6. M. Chanda, K. F. O'Driscoll, and G. L. Rempel, "Sorption of Phenolics and Carboxylic Acids on Polybenzimidazole," Reactive Polymers 4, 39 (1985).

7. J. Ungar, Dianex Systems, personal communication, November 1990. 

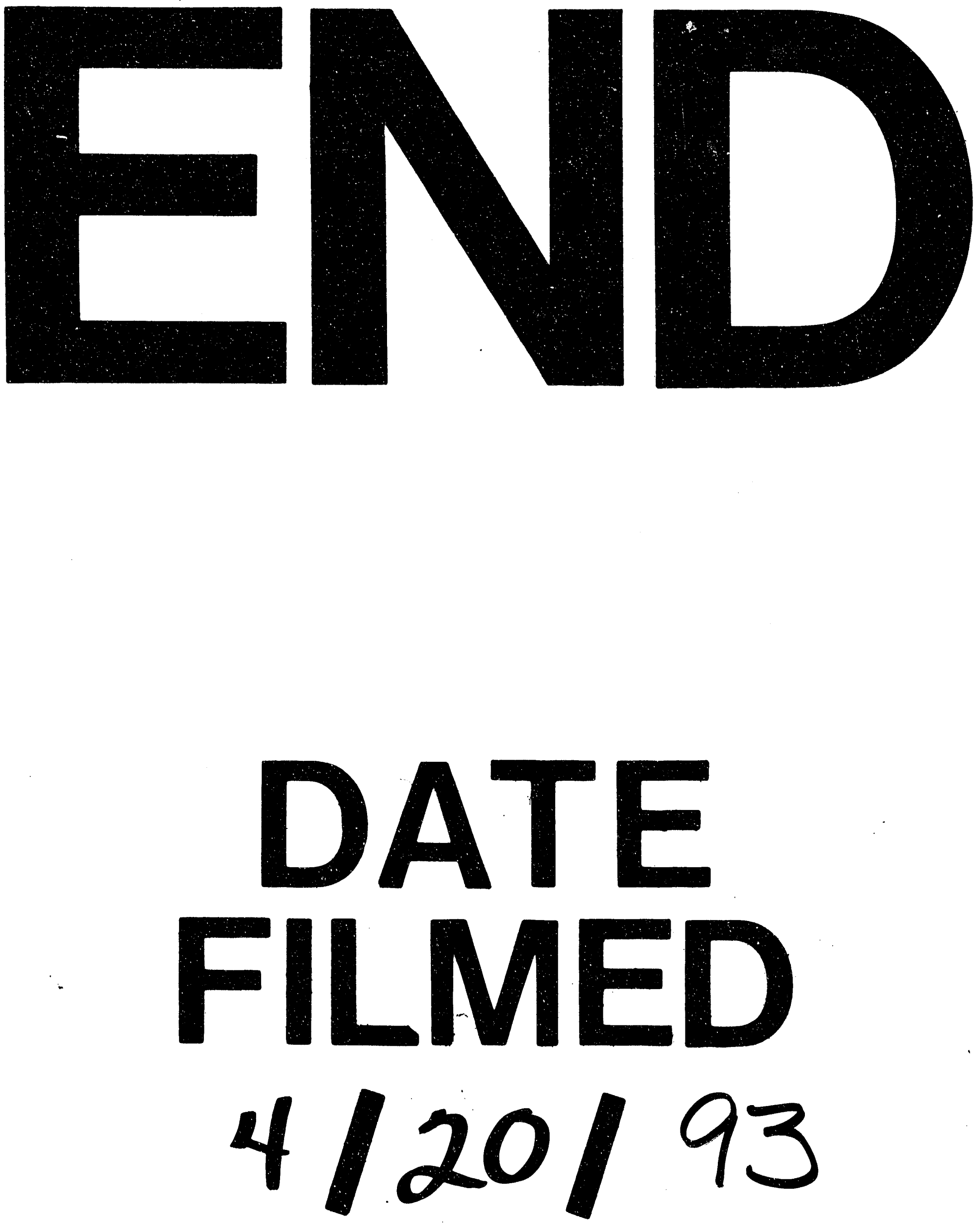

1 
